\title{
La vinculación para la transferencia de conocimiento, científico, tecnológico e innovación, aplicado al sector mueblero de Jalisco
}

Evelio Gerónimo Bautista*

\section{RESUMEN}

El objetivo del presente artículo es explicar la transferencia de conocimiento científico, tecnológico e innovación en el sector mueblero, vinculado con las universidades y con el gobierno del estado de Jalisco. Los resultados muestran que las empresas se han vinculado informalmente con la UDG, el ITESM y el ITESO. Éstas cuentan con una infraestructura tecnológica adecuada para la transferencia. El 31\% de las empresas aprovechan el conocimiento científico tecnológico de las universidades a través de programas de vinculación como consultorías, servicio social, etcétera. La metodología utilizada fue mixta; se encuestaron a 290 empresas, se entrevistaron a siete representantes de universidades y dos representantes de organismos gubernamentales; los resultados obtenidos fueron analizados en dos softwares: SPSS y Atlas TI.

Palabras clave: transferencia, innovación, universidades, organismos gubernamentales y sector mueblero.

Clasificación JEL: I23, L60, O31.

* Profesor-investigador, Universidad del Valle de Atemajac, Guadalajara, México. Correo-e: evelio.geronimo@univa.mx Orcid: https://orcid.org/0000-0001-6795-0404 


\section{ABSTRACT}

\section{The linkage for the transfer of scientific and technological knowledge and innovation, applied to the furniture sector of Jalisco}

The objective of this article is to explain the transfer of scientific, technological and innovation knowledge in the furniture sector, linked to universities and the government of the state of Jalisco. The results show that the companies have been informally linked with the UDG, ITESM and ITESO. These have an adequate technological infrastructure for transfer. $31 \%$ of companies take advantage of the scientific and technological knowledge that universities have through linkage programs such as consultancies, social service, etc. The methodology used was mixed, 290 companies were surveyed, 7 representatives of universities and two representatives of government agencies were interviewed, analyzed in SPSS and Atlas TI.

Keywords: transfer, innovation, universities, government agencies and the furniture sector.

JEL Classification: I23, L60, O31.

\section{INTRODUCCIÓN}

El objetivo principal del artículo es explicar la transferencia de conocimiento científico, tecnológico e innovación al sector mueblero, vinculado con las universidades y con el gobierno del estado de Jalisco.

Uno de los factores claves de éxito en una competitividad, desarrollo y cambio regional es la vinculación de varias empresas con la academia, órganos de gobierno y despachos de asesorías, en donde las empresas funcionan como proveedoras en representación de todas aquellas proveedoras y diseñadoras de la academia en diferentes ámbitos. La facilidad de la transferencia de conocimiento científico, tecnológico e innovación tiene que ver con aspectos de trabajo en conjunto a través de convenios de vinculación, gestión de tecnología e innovación de las empresas como componentes claves para su crecimiento y permanencia en el tiempo. Es importante considerar perfiles para la dirección de empresas de cualquier tamaño para realizar actividades operativas, administrativas, directivas $\mathrm{y}$, sobre todo, la gestión del conocimiento 
científico, tecnológico e innovación a través de un acuerdo de colaboración y/o vinculación para una exitosa transferencia.

En este trabajo se explican los elementos necesarios para llevar a cabo una transferencia de conocimiento científico, tecnológico e innovación entre las universidades y el gobierno con la industria del mueble. Se toman en cuenta las mejores prácticas de otros países y de México sobre el tema.

La pregunta principal que se plantea en este trabajo es la siguiente: ¿Cómo se aplica la transferencia de conocimiento científico, tecnológico e innovación -implícita en los procesos de vinculación generados por las IES-al sector productivo de muebles de Jalisco? Se responderá esta cuestión al largo del documento.

\section{ANTECEDENTE Y JUSTIFICACIÓN DEL ESTUdiO}

La presente investigación es parte de un proyecto de tesis doctoral en la que se engloban aspectos como formación de talento humano, apoyos económicos y vinculación como plataforma para realizar trabajo de colaboración a través de acuerdos y convenios entre instituciones académicas con empresas y gobierno, con intervención de la sociedad civil. Este trabajo nace de la carencia de información, principalmente de la industria y academia, debido a que es tradicional, aunque se refleja la inversión en tecnología intermedia, misma que se presenta en los resultados de esta investigación, así como en los apoyos recibidos por parte del gobierno federal y estatal.

Es crucial dar a conocer el eje de transferencia de conocimiento científico, tecnológico e innovación para que se trabaje y se fortalezca entre la academia y la industria, dado que otras industrias en desarrollo y desarrolladas que han realizado esta actividad han tenido éxito, debido a que se comparte conocimiento generado desde las universidades para apoyar al desarrollo de la industria.

\section{TRANSFERENCIA DE CONOCIMIENTO CIENTíFICO, TECNOLÓGICO E INNOVACIÓN}

\subsection{Transferencia de conocimiento científico \\ El conocimiento se ha convertido en el principal recurso productivo, por lo que las organizaciones han comenzado a valorar su importancia,}


ya que en la sociedad representa la etapa más avanzada del proceso de transformaciones significativas impulsadas por la globalización (García, 2004).

La transferencia de conocimiento es inherente a la educación desde su generación, desarrollo, difusión y transferencia (Touriñán, 2019), por eso su importancia. El conocimiento se produce a través de la triple hélice y se transfiere por cuarta y quinta hélice (Bautista, 2015), lo que significa que esta transferencia tiene sus etapas; primero se vinculan universidad-industria-gobierno a través de convenios de colaboración patentes y licencias, proyectos de financiación y desarrollo empresarial. Al respecto, Touriñán (2019) muestra en su investigación los resultados que genera la transferencia de conocimiento, como empresas de nueva creación, nuevos contratos de investigación, acuerdos sobre licencias, mejora de emprendimiento, mejora de formación y de la investigación colaborativa. Ante los cambios vertiginosos de la globalización, obliga a las instituciones a gestionar la capitalización del conocimiento (Viale y Etzkowitz, 2010), toda vez que la inserción eficiente de la institución universitaria en la sociedad del conocimiento requiere un cambio en la concepción tradicional de la organización, que implica su participación directa en el desarrollo regional y nacional como un ente social en el ecosistema emprendedor de las empresas (Guerrero y Santamaría, 2020). Desde hace más de 15 años, García (2004) propone algunos objetivos para una estructura organizacional que facilite la gestión del conocimiento y la actuación de una universidad emprendedora:

- Desarrollar un plan de educación activa.

-Instrumentar un enfoque académico exigente, estimulante y dinámico.

- Crear un ambiente institucional adecuado para la realización de actividades de innovación y desarrollo tecnológico.

Estos objetivos están orientados a integrar las actividades universitarias al sector productivo; funciona como transferencia y aplicación de conocimiento, además de permitir vincular sus demás funciones sustantivas como universidad comprometida con las necesidades y requerimientos de innovación e investigación y desarrollo tecnológico con los sectores productivos.

De acuerdo con Arechavala (2010), la universidad emprendedora se da principalmente en países europeos. En México, en cambio, las autoridades universitarias y el sistema de educación superior todavía 
están lejos de entenderlo; debe haber un equipo directivo capaz de articular un programa efectivo para desarrollar capacidades específicas en la sociedad del conocimiento para el fomento de la innovación y la transferencia.

Al respecto, Arechavala propone algunos elementos para la gestión de la capitalización del conocimiento en la era de la sociedad del conocimiento:

a. Capacidad para vincularse con la industria y de responder a sus necesidades en tiempos relevantes.

b. Capacidad legal para procesar convenios de transferencia y comercialización de conocimiento.

c. Capacidad para gestionar eficientemente y con visión de largo plazo derechos de propiedad intelectual en contratos de licenciamiento de tecnología que resulten en las mayores ganancias para todas las partes involucradas.

d. Capacidad de incentivar entre sus investigadores y estudiantes el contacto con empresas y el trabajo de investigación aplicada e incluso de aplicación industrial

e. Capacidad de identificar, formar y organizar talento empresarial y de negocios para la explotación de tecnologías desarrolladas en la universidad.

f. Capacidad para organizar y apoyar con recursos suficientes la operación de una oficina de transferencia de tecnología.

g. Capacidad de movilizar el conocimiento de los profesores y estudiantes en labores de apoyo a la industria, a organizaciones no gubernamentales y organizaciones públicas (2010: 10).

Estos elementos se aplican de acuerdo al compromiso de la universidad en su contexto social y económico, en la medida que ésta defina con claridad los mercados de conocimiento en los que quiera participar; lo más importante es que se tenga la capacidad para realizar este tipo de actividades para poder medir y capitalizar el conocimiento.

Según investigaciones de Viale y Etzkowitz (2010), la capitalización del conocimiento ocurre cuando éste genera una economía de valor añadido; la generación puede ser "directa", cuando se vende el conocimiento, o "indirecta", cuando permite la producción de algunos bienes materiales o servicios que se venden en el mercado; es decir, el valor añadido directo se vende y el valor añadido indirecto juega en el mercado tipo bienes y servicios. En otras palabras, el modo directo 
comprende la venta del know-how, esto es, la venta de la propiedad intelectual, como en el caso de las patentes, derechos de autor o de enseñanza. El modo indirecto comprende las formas en que la organización y el conocimiento se materializan en bienes o servicios.

Por eso, no es fácil identificar la transferencia de la capitalización del conocimiento, pero viene de la interacción entre el ámbito académico y el empresarial, donde son más débiles cuando trabajan solos; cuando interviene el gobierno, ya sea regional o estatal, es posible que el desarrollo económico sea eficientemente más efectivo. El gobierno juega un papel importante al aportar capital de riesgo para las industrias y ofrecer incentivos a través de becas. No se puede dejar de percibir la triple hélice, en donde parece ser el jugador de un anticíclico de innovación o co-evolutivo que Geels (2006) refiere en su texto sobre los sistemas de aviación, al referirse a un modelo que garantiza el nivel óptimo entre la interacción universidad-empresa a través de la intervención del gobierno para generar una transferencia exitosa y, en consecuencia, políticas públicas (Viale y Etzkowitz, 2010).

\subsection{Transferencia tecnológica}

La transferencia de tecnología es una actividad crucial para las universidades y para la agenda de los gobiernos como política pública hacia la industria, con la finalidad de fortalecer las economías locales (Debackere y Veugelers, 2005), actividades de las que pueden resultar incentivos y conocimientos nuevos. La globalización e internacionalización exige a las universidades e instituciones de educación superior transferir los conocimientos científicos, tecnológicos y de innovación que se generan en sus instalaciones a los sectores productivos, sociales y públicos. En el caso de Estados Unidos se creó la ley Bayh Dole Act, nacida en los años 80 , que fundamenta la transferencia de tecnología. La literatura especializada se refiere a ella como ejemplo para explicar y aplicar la transferencia de tecnología entre universidades e industria; por ello se toma como referencia en este trabajo para explicar la transferencia de conocimiento CTI.

Es importante mencionar que existen muchas partes interesadas en realizar y aplicar la transferencia de tecnología, desde los investigadores, los famosos Technology Transfer Offices (TTO), instituciones privadas, entre otras (Anderson et al., 2007). En este sentido, para la economía de EE.UU. ha sido prioritaria la transferencia de tecnología 
porque fortalece las empresas locales, que incluyen servicios de ingeniería, reingeniería, entre otras. Por otro lado, en EE.UU. han sido criticadas muchas universidades porque les resultó difícil generar y transferir la tecnología suficiente hacia la industria; no obstante, la ley Bayh Dole Act ha sido una plataforma para que las universidades norteamericanas se vinculen con el sector productivo y tranfieran las tecnologías que requiere el sector para el desarrollo de sus productos, procesos, entre otros. Esta práctica se ha llevado a cabo en muchas universidades -MIT, Stanford y Harvard- las cuales han creado centros de transferencias de tecnologías para difundir licencias o patentes a la industria (Siegel et al., 2003).

En este tenor, Lai (2011) documentó que en Taiwán la comercialización de la tecnología y la distribución de las licencias de las tecnologías juegan el rol más importante en la transferencia desde la universidades. En este país asiático se toman en cuenta las capacidades de los profesores que generan tecnología, por lo que se llega a la conclusión de que los profesores reciben más incentivos que otros por los avances tecnológicos.

El rol de los profesores, incluso en las agendas de los gobiernos en Europa, para la transferencia de tecnología es clave para que las empresas se conviertan en competitivas tanto local como regional e internacionalmente. De esta manera, el Kid, conocido también como Know How, sólo transporta al profesor-investigador y lo transfiere hacia la industria. Debackere \& Veugelers (2005) afirman que la transferencia científica y tecnológica han sido prioritarias en las agendas políticas para generar actividad económica; puntualizan que las oficinas de transferencia de tecnología (OTT) son una organización desentralizada que involucra grupos de investigadores que generan desde patentes hasta creación de spin off, es decir, "empresas de nueva creación".

En este sentido, Wright et al. (2008) explican que existen universidades enfocadas en la clase media, que contribuyen a cambios sustanciales en la industria a través de conocimiento tácito y codificado, es decir, generan empresas de nuevas licencias, patentes, asesorías y consultorías. Estas universidades tratan de transferir conocimiento científico y tecnológico a la economía local, porque para las empresas es más importante tener cerca una institución que solucione sus problemas, toda vez que es más difícil que una universidad de clase mundial aterrice las micro, pequeñas y medianas empresas. 
Estos claros ejemplos se pueden tomar en cuenta para la realización de transferencia de tecnología en regiones como México. Otro de los puntos importantes que hay que considerar sobre la transferencia de tecnología es que muchas veces se da formal e informalmente, hecho que ha documentado Siegel et al. (2003) y Lai (2011), quienes regularmente se han basado en una ley; no obstante, autores como D'Este y Patel (2005); D'Este y Iammarino (2009) y D'Este et al. (2014) se enfocan en documentar las transferencias informales, en las que no se utiliza ningún contrato para realizar este tipo de actividades, es decir, el involucramiento de científicos y tecnólogos en la industria se da bajo ninguna restricción de una ley.

Por consiguiente, la transferencia de tecnología y del conocimiento en general, ya sea que se haya adquirido o transferido formal e informalmente, se capitaliza hasta convertirse en un producto o servicio a través de la innovación. Incluso la transferencia de tecnología tiene que ver con la difusión de conocimiento por medio del desarrollo productivo y la innovación (Montiel et al., 2020).

De acuerdo con las investigaciones de Londoño et al. (2018), existen varios modelos que se pueden utilizar para la transferencia de conocimiento CTI, como modelos de apropiabilidad, modelo de difusión, modelo de utlización del conocimiento, modelo lineal, modelo no linea de la triple hélice, modelo dinámico y modelo latinoamericano; todos ellos tienen que ver con un proceso de innovación.

\subsection{Innovación}

La Real Academia Española (2020) define la innovación como "mudar o alterar algo, introduciendo novedades"; este concepto introduce una novedad por medio de elementos y/o procedimientos precisos y objetivos especializados en el mueble, donde participan actores clave que trabajan como sistema. Aproximadamente hace cuatro décadas, Lundvall (1992) y Nelson (1993) desarrollaron el concepto de Sistema Nacional de Innovación, evidentemente institucional, que ha jugado un papel crucial en la teorización del proceso tecnológico, toda vez que los cambios tecnológicos y organizacionales están descritos, por lo que las instituciones juegan un papel importante en el desarrollo económico como un sistema. Por ello, el concepto habla de la integración de múltiples actores para el desarrollo y flujo de circulación del conocimiento, así como las instituciones, ya que la articulación de múltiples actores 
resuelve problemas al poner en acción los agentes y los efectos en la interacción del aprendizaje. La innovación no depende de un solo actor sino de un sistema de actores que trabajan bajo una red de colaboración desde las dimensiones macro, meso y microeconómicas para la generación de aprendizajes conjuntos organizacionales, sociales y territoriales; esto es lo que ha funcionado en el desarrollo de las economías de países emergentes.

El concepto de Sistema Nacional de Innovación será útil para evaluar la densidad de vínculos y su continuidad nacional en el sector mueblero. La estructura institucional del Consejo Mexicano del Mueble y Decoración contribuye a desarrollar políticas públicas para regular el desarrollo económico de la industria mueblera y el involucramiento de otras instituciones (universidades, centros públicos de investigación, ONG, etc.)

Los sistemas de "innovación" han traído nuevos puntos de vista a la escena, pero a partir de los años 90, con las aportaciones de los autores referidos, se crea la Triple Hélice de Etzkowitz y Leydesdorff (1995), modelo innovador en países desarrollados y recientemente en países de América Latina, donde la innovación es resultado de la participación de la universidad-empresa-gobierno. Expertos como Rickne et al. (2013) definen la innovación como un "sistema regional de innovación en donde surge de la complejidad de los procesos de conocimiento en la interacción social y los contactos personales que son importantes en los procesos de aprendizaje y por lo tanto la proximidad geográfica es tomada en cuenta dentro de una región que ofrece el mecanismo óptico para la transferencia e intercambio de conocimientos".

En este sentido, la innovación se puede dar en los ámbitos global, empresarial e institucional, susceptibles de por sí a ser innovadores. Las fortalezas se dan a través de sistemas de innovación; es decir, por medio de la participación de la universidad y las empresas ubicadas en una región geográfica. Se considera la participación del gobierno local como apoyo a estas instituciones para poder dar un paso hacia el crecimiento y el desarrollo regional, ya que aporta recursos financieros para la compra de tecnología de punta, pilar en la generación de la innovación. La reflexión sobre los procesos de innovación se puede implementar en la industria mueblera de Jalisco con acciones innovadoras y resultados exitosos en otras naciones, que en la actualidad son de las más competitivas comercialmente en el mundo. 
$\mathrm{Al}$ respecto de las instituciones dedicadas a la generación de conocimiento, Ahonen y Hämäläinen señalan: "no son los únicos generadores de conocimiento. Muchas grandes compañías internacionales mantienen grandes departamentos de $\mathrm{I}+\mathrm{D}$, poseen un gran número de patentes y son a menudo capaces de contratar a los mejores cerebros de una generación con incentivos financieros más altas que las universidades públicas. El sector público en su conjunto está sufriendo de cortes financieras continuas y la disminución de los recursos humanos. No puede absorber innovaciones producidas por muchas razones, una de ellas es el sistema muy rígido de la contratación pública, que originalmente significaba para hacer el uso del dinero de los contribuyentes más transparente y sus usuarios una mejor rendición de cuentas, un buen objetivo que llevó a una raza no deseado, si se nos permite decirlo" (2012: 17).

No obstante lo anterior, en México hay más Pymes que compañías grandes, por lo que lo afirmado por Ahonen y Hämäläinen no es posible. Empero, subrayan que la innovación está en discusión; por ejemplo, la triple hélice es incapaz de incorporar una cantidad de innovadores o de igualdad en el sistema; sin embargo, es la base para la generación de innovación, aunque Ahonen y Hämäläinen mencionan que se necesita algo diferente, como la "cuádruple hélice", establecida a partir de la suposición de que una Asociación Civil (AC) es el elemento faltante para la innovación, o bien, la incorporación de otros actores heterogéneos.

\section{Metodología}

La estrategia utilizada es de tipo exploratoria, con enfoque mixto; además, es una investigación aplicada como lo señala el Conacyt en su página web. Por otro lado, Creswell (2014) y Hernández et al. (2010) afirman que hay tres tipos de métodos de investigación académica en la educación superior: cuantitativa, cualitativa y enfoque mixto, utilizada en esta investigación. El método mixto es utilizado desde 1959 por Campbell y Fiske, quienes lo denominaron Matriz multimétodo, para revisar múltiples enfoques en la obtención datos en un estudio (Creswell, 2014). Se encuestaron 290 empresas, incluyendo organismos empresariales, siete universidades y dos organismos gubernamentales; 
los resultados obtenidos fueron analizados en dos softwares: SPSS y Atlas TI.

La investigación se explica a través del procesamiento y análisis de la información mediante instrumentos de acopio, como encuestas y entrevistas semi-estructuradas, aplicadas a diferentes expertos en instituciones de educación superior, empresas muebleras y organismos empresariales, y organismos gubernamentales. Con base en teorías y mejores prácticas, se analizaron las dimensiones y los ejes estratégicos, y se hizo hincapié en la transferencia de conocimiento científico, tecnológico y de innovación.

FIGURA 1

DIMENSIONES Y EJES ESTRATÉGICOS

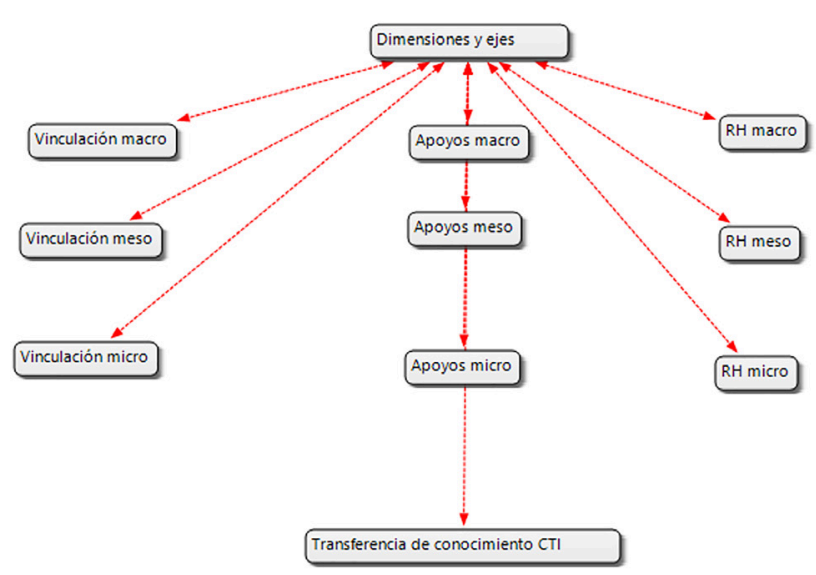

Fuente: elaboración propia.

Las empresas muebleras participantes en este estudio fueron analizadas como micro, pequeñas, medianas, así como instituciones de educación superior de Jalisco y organismos gubernamentales (Tabla 1). 
TABLA 1

TAMAÑO DE EMPRESA

\begin{tabular}{ccc}
\hline Tamaño de empresa & Frecuencia & Porcentaje \\
\hline Micro & 123 & $42 \%$ \\
\hline Pequeña & 120 & $41 \%$ \\
\hline Mediana & 34 & $12 \%$ \\
\hline Grande & 13 & $4 \%$ \\
\hline Total & 290 & $100 \%$ \\
\hline
\end{tabular}

Fuente: elaboración propia.

Las empresas muebleras representan una muestra importante de participación en los procesos de vinculación para la transferencia de conocimiento científico, tecnológico e innovación a través de los académicos y organismos gubernamentales. Se observa que el sector industrial mueblero está compuesto por $42 \%$ empresas micro, $41 \%$, pequeñas, $12 \%$, medianas y sólo $4 \%$ son grandes empresas muebleras.

\section{RESULTADOS DE LA INVESTIGACIÓN}

Los esquemas de colaboración entre público-privadas han sido y siguen siendo importantes para el avance del crecimiento económico de un país, tal como lo mencionan autores internacionales. En Jalisco existe un avance de colaboración y vinculación entre las empresas muebleras del estado, las universidades y los organismos gubernamentales, donde básicamente el avance de vínculos ocurre a nivel meso y micro. No se ha logrado tener una vinculación a nivel macro, debido a la falta de políticas de CTI, que aún están en construcción.

En la Figura 2 se pueden apreciar los vínculos y las redes de colaboración con las asociaciones y cámaras empresariales (Afamo, Afamjal, Careintra, Canaco Tlaquepaque, CS Muebles AC, Canacintra de México), consecuentemente se vinculan algunas universidades, como CUAAD, CUCEA, CUCEI, CUCIENEGA; UP, UTZMG, UAG, ITESM e ITESO, y otras como UVM, UNIVER, UNIVA, Tecnológico de Ocotlán y CETI Ocotlán y, desde luego, algunos organismos gubernamentales (Coecytjal, Fojal, Impi y Conacyt). Estos vínculos han permitido la realización de acciones en conjunto; los apoyos han 
facilitado las asociaciones entre las empresas muebleras, asesorías y consultorías sobre las áreas básicas, incluso legales, ofrecidas por las universidades, así como programas de crédito o financiamiento de los organismos gubernamentales.

FIGURA 2

RED DE COLABORACIÓN ENTRE EMPRESAS MUEBLERAS CON LAS UNIVERSIDADES Y LOS ORGANISMOS GUBERNAMENTALES

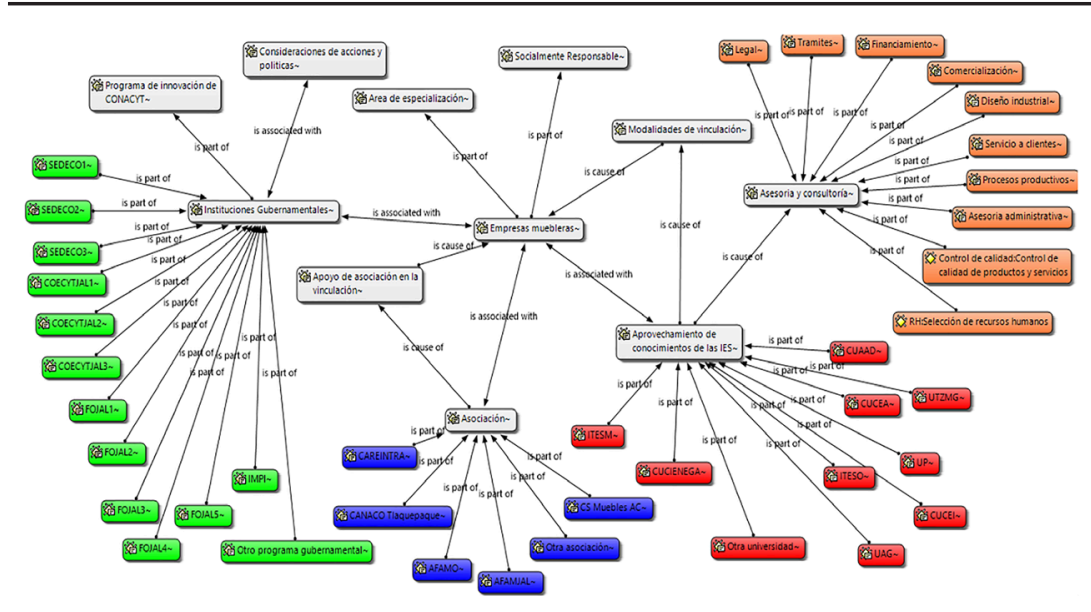

Fuente: elaboración propia.

La transferencia de conocimiento científico, tecnológico e innovación constituye entre los agentes heterogéneos, ya que demuestra la cantidad de conocimiento aplicado en las empresas muebleras. En este caso se puede apreciar el fortalecimiento de las empresas a traves de esta transferencia. 
GRÁFICA 1

FORTALECIMIENTO DE LAS EMPRESAS CON LAS IES

\begin{tabular}{|c|c|c|c|c|c|c|}
\hline & \\
\hline
\end{tabular}

Fuente: elaboración propia.

El aprovechamiento del conocimiento científico y tecnológico en las empresas muebleras representa 31\%; asimismo, 53\% representa los activos tangibles e intangibles en la inversión, como maquinaria o patentes; $11 \%$ realiza alguna investigación o desarrollo experimental e innovación en colaboración con las universidad; por último, se solicitó información sobre la introducción de innovaciones con la participación de las universidades durante el periodo 2006-2014, área que representa sólo $7 \%$, esto quiere decir que durante ocho años no se generaron innovaciones en las empresas muebleras en conjunto con las universidades.

Asimismo, a través de las empresas se obtuvo información sobre la inversión en activos, productos tangibles e intangibles, detonantes de la transferencia de conocimiento científico y, consecuentemente, la generación de innovación. La inversión en maquinaria y equipo en las microempresas tiene un activo básico para su operación que representa $18 \%, 6 \%$ en software y base de datos, $16 \%$ de inversión en maquinaria y equipo, $6 \%$ en investigación y desarrollo, y $4 \%$ en uso de software y 
base de datos; respecto a las medianas y grandes empresas casi no se han reflejado grandes cantidades de inversión en sus instalaciones. Es importante resaltar que el uso de derechos de propiedad de la marca refleja $4 \%$ en todos los tamaños de las empresas, lo que significa que tiene pocas actividades de inversión en el registro de sus productos.

GRÁFICA 2

INVERSIÓN EN ACTIVOS

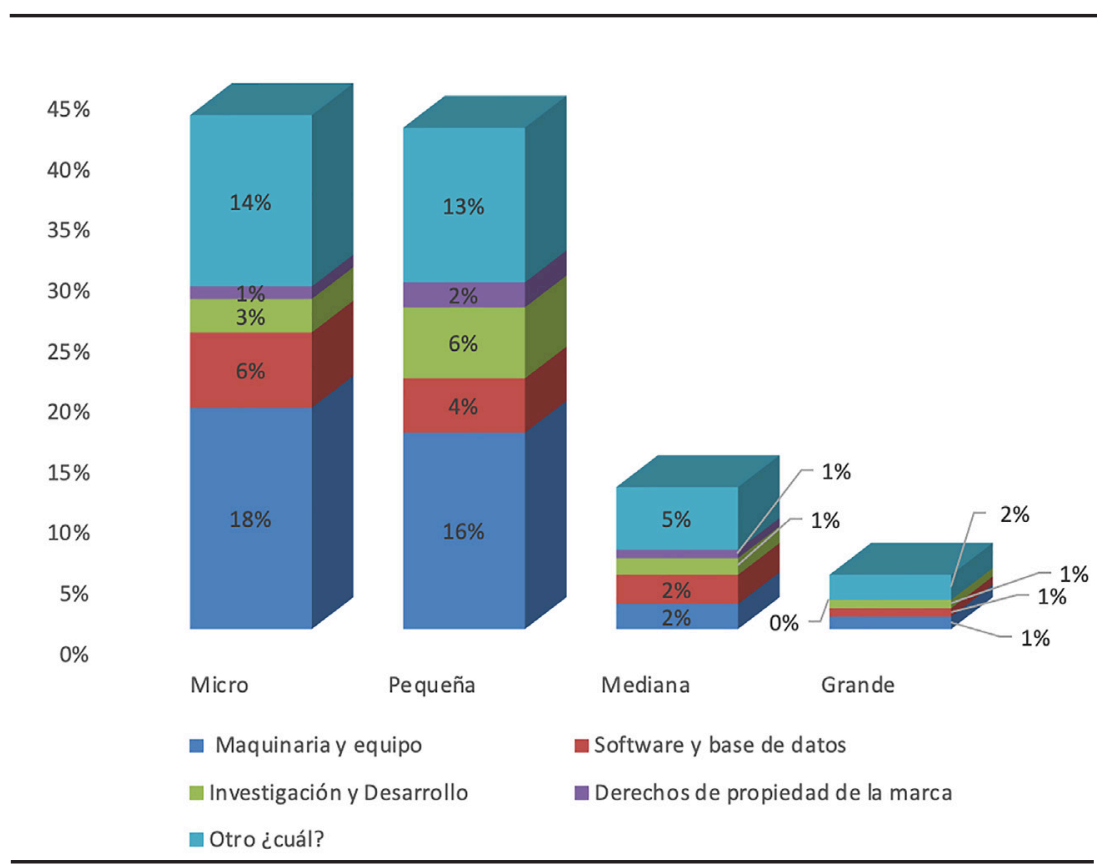

Fuente: elaboración propia.

Unas de las preguntas que se realizó a las empresas fue qué tipo de innovación habían consolidado con las universidades; $72 \%$ corresponden al rubro "Otro ¿Cuál?”, es decir, no ha habido innovaciones; únicamente $3 \%$ de las micros, $3 \%$ de las pequeñas y $1 \%$ de las medianas realizaron innovación organizacional o mercadotecnica; otros $3 \%$ de las micros y $3 \%$ de las pequeñas realizaron innovación organizacional o en mercadotecnia e innovación de productos y procesos. Por otro lado, en lo que corresponde a innovación únicamente de productos o procesos, las micro representaron $2 \%$, las pequeñas, $6 \%$, y las medianas, 3 por ciento. 
En cuanto al tipo de colaboradores con los que cuentan las empresas, base para generar innovaciones, $52 \%$ cuenta con enfoque de innovación; $6 \%$ corresponde a innovadores informales que colaboran en las micros; $9 \%$ en las pequeñas y $2 \%$ en las medianas. En las empresas grandes, los informales colaboradores no se reflejan casi nada: micro, $6 \%$, pequeñas, $5 \%$, mediana, $2 \%$ y grande, 1 por ciento.

GRÁFICA 3

RESULTADOS DE LA INVERSIÓN EN INNOVACIÓN EN LAS EMPRESAS MUEBLERAS

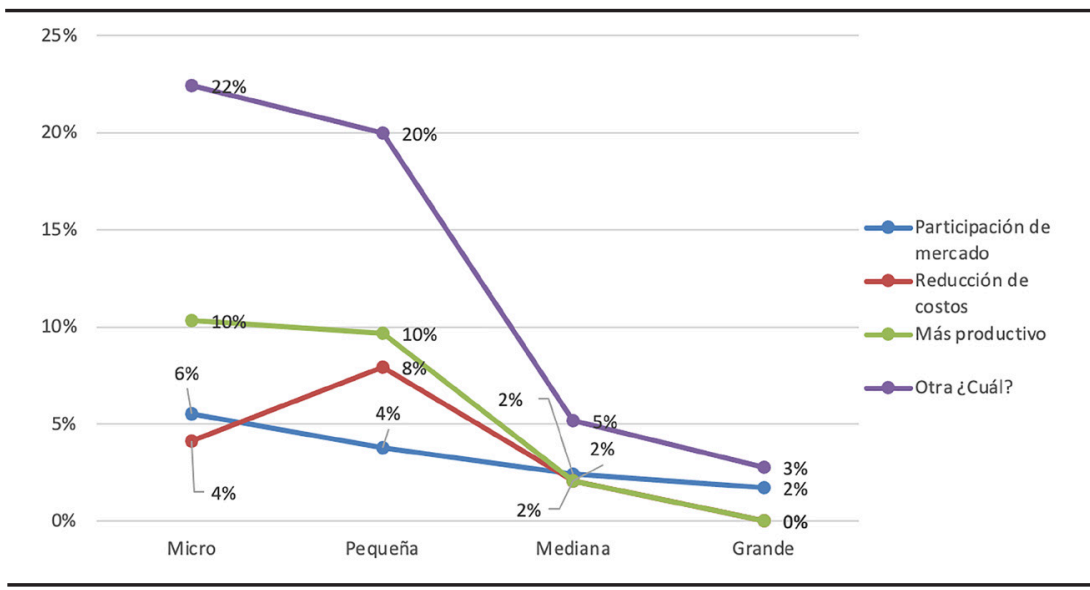

Fuente: elaboración propia.

Se evidencia una tendencia respecto al rubro "Más productivo" en los resultados de inversión en innovación: 10\% en las microempresas; $10 \%$ en las pequeñas, sólo 2\% en las medianas; la "Participación de mercado" representa $6 \%$ en las micros, $4 \%$ en las pequeñas y $2 \%$ en las medianas. Por otro lado, la inversión en innovación ayudó a las empresas a reducir costos, sobre todo en las pequeñas empresas con $8 \%$, mientras que en las micro sólo 4 por ciento. Por último, $50 \%$ no han realizado inversión para la innovación.

Algunas de las universidades de Jalisco con las que se han vinculado las empresas muebleras, en términos de aplicación de los conocimientos científicos y tecnológicos, han logrado dos patentes, que se encuentran en trámite para su aprobación. Todos los derechos se ceden a las empresas beneficiadas, además de que se ofrecen capacitaciones para la protección de marcas hasta su registro. Lamentablemente no todas las universidades cuentan con una adecuada estructura -tanto 
administrativa como de infraestructura- para la transferencia de conocimiento científico y tecnológico, aunque ya se cuenta con el centro de diseño, que alberga equipamiento para transferencia.

Respecto a las actividades de transferencia en algunas universidades privadas, la investigación es aplicada por declaración y naturaleza para crear un impacto positivo en el corto y mediano plazo, para finalmente llegar a ser un producto o un servicio para apoyar a la creación de una empresa como estrategia de innovación. Cuentan con oficinas de transferencia tecnológica (OTT) para gestionar la investigación producida en la institución, así como la gestión de protección de los productos para transferirlos a las empresas; es decir, la OTT detona proyectos que pueden ser desarrollados por los centros o los grupos de investigación, con fondos del gobierno u otros. Asimismo, estas universidades cuentan con estructura administrativa y personal especializada; por ello las aportaciones de conocimiento científico y tecnológico son de investigación aplicada, nuevos procesos de innovaciones, algunos instalados en las empresas y otros comercializados y publicados.

Muchas instituciones de educación superior están a la vanguardia por las plataformas educativas, redes sociales, etc., que constituyen una forma de trasmisión de conocimiento en el proceso de enseñanza. Continuamente las universidades desarrollan infraestructura tecnológica, misma que aplican en las aulas, a través de redes internas, sistemas y servicios, recursos con los que cuentan. Por otro lado, están en constante formación para adquirir conocimientos mediante educación continua, diplomados, talleres, entre otras actividades, que se realizan para el sector productivo o público. Es una manera de aplicar sus conocimientos y fortalecer la relación entre gobierno, universidad y el sector empresarial.

\section{Discusión DE RESULTADOS}

En la Universidad de Guadalajara se encuentra el departamento de Madera, celulosa y papel, que durante un tiempo estuvo apoyando a la cadena mueblera; no obstante, debido a la poca respuesta de la industria mueblera y a la demanda por parte de los empresarios, el departamento se replegó a otras actividades de celulosa y papel. Es importante explicar que es difícil que los investigadores de cualquier universidad 
puedan realizar actividades de desarrollo para la cadena productiva o de procesos tecnológicos.

En términos generales, las principales instituciones que cuentan con infraestructura adecuada para la transferencia de conocimiento científico y tecnológico, públicas o privadas, son la UDG, Tecnológico de Monterrey, UP, ITESO, UNIVA y la UAG. En el caso de la UDG, se ha invertido en sus diferentes centros para construir laboratorios y espacios; la Universidad Panamericana, por su parte, cuenta con áreas de muebles, para las que adquirió equipos y maquinaria operados por ingenieros para desarrollar prototipos. Asimismo, el TEC de Monterrey tiene laboratorios y centro de diseño, mientras que el ITESO cuenta con centro de diseño y aulas equipadas para ofrecer capacitaciones al sector empresarial.

De acuerdo con lo anteior, se han hecho propuestas para el mejoramiento de las TIC a través de programas como ERP, sistema de software para control que engloba áreas básicas como manufactura, finanzas, contabilidad y producción, administrativas y de comercialización, así como proyectos estratégicos como parques industriales, creados a través de lay out completo, y manufactura esbelta ad hoc, así como gestiones de la innovación y programas de diseño en la empresa.

Las aportaciones de conocimiento científico, tecnológico e innovación para contribuir a la competitividad, aplicadas en muy pocas empresas, son: mejoras de procesos y estudios de mercados; no obstante, no se han implementado proyectos de desarrollo tecnológico que hayan beneficiado a la industria mueblera. Los estudios de mercado realizados por la Universidad Tecnológica de la Zona Metropolitana de Guadalajara (UTZMG), en conjunto con las empresas muebleras y gobierno se enfocaron en la generación de sistemas de control de costos, nóminas, sistemas simplificados, control de inventarios, relaciones con clientes para la exportación.

Desde el punto de vista gubernamental, no cabe duda que las universidades generan conocimiento científico y tecnológico, pero se quedan con él, pocas veces lo transmiten y aplican a la industria mueblera. Por ejemplo, se ha generado conocimiento CTI en CUCIENEGA, ubicado en Ocotlán, Jalisco, región Ciénega, pero lamentablemente su implementación salió mal, ya que el compromiso de cada actor no se dio de la mejor forma; esto es, se se salió del control de los académicos que lo dirigían. Por lo tanto, el empresario no quedó contento. Otro de 
los casos fue entre empresas que fabrican equipales en conjunto con el centro universitario del sur de la Universidad de Guadalajara. Las empresas optimizaron $20 \%$ de tiempo al fabricar una parte del mueble equipal ("la barita que sostiene el equipal que va cruzada y que se llama estaca; a esa estaca se le hace un tipo punta como un cuello, que es justamente en donde va agarrar el hilo o cuero con lo que se marra la base del equipal"). La universidad participó con el desarrollo de una máquina para que los equipaleros siguieran su proceso artesanal.

Por otro lado, existen organismos gubernamentales que financian recursos económicos para el desarrollo y fortalecimiento de las capacidades productivas de las empresas, pero lamentablemente desconocen que las universidades generan y aplican conocimiento, además de que cuentan con estructura adecuada para la transferencia de conocimiento científico y tecnológico para fortalecer los procesos de innovación en la industria mueblera de Jalisco.

Tampoco se conoce algún investigador que haya proporcionado desarrollo específico a la cadena productiva. El mayor logro conseguido fue la unión de los diferentes organismos durante casi 8 años; lamentablemente es un sector desarticulado, debido a que hay varias cámaras o asociaciones empresariales que lo representan. Lo ideal es conjuntarlo para que trabajen por un único objetivo; para ello se han hecho varios talleres, como planeación estratégica, para detonar proyectos enfocados a la comercialización, tanto local como internacional.

En términos generales, la transferencia de conocimiento científico, tecnológico e innovación es escasa. De acuerdo con los resultados, muchas universidades sí cuentan con infraestructura, pero no lo aplican o muchas empresas no se acercan a las instituciones para buscar la manera de apoyarse con sus instalaciones

\section{CONCLUSIONES}

La industria manufacturera de muebles ha vivido procesos de transformación de empresarios con un perfil más tradicional, con conocimientos de manufactura poco actualizados y tecnificados, como los implementados en países considerados muy competitivos a nivel mundial. Por ello, la inversión en este rubro y una mayor participación en proyectos conjuntos entre los sectores productivos y académicos es imperativa, así como el apoyo del gobierno para la asignación de fondos públicos 
para el fortalecimiento de la planta productiva con fines de innovación y competitividad.

Se concluye que sí existe infraestructura tecnológica en las universidades e IES públicas y privadas para la transferencia de conocimiento CTI; sin embargo, los retos y desafíos de la vinculación universidadempresa-gobierno para el presente milenio son enormes, considerando la fragilidad o volatilidad de las finanzas de las empresas, instituciones u organismos.

Dentro de los procesos de vinculación para la innovación entre los agentes heterogéneos que fortalecen al sector mueblero del estado de Jalisco, se puede afirmar que sí existen avances de vinculación entre las universidades (UDG, ITESM, ITESO) con los organismos empresariales del mueble (Afamo, Afamjal, Careintra y Canaco Tlaquepaque), incluso con algunas empresas muebleras directamente. El problema es que esta vinculación ha sido informalmente; es decir, los académicos y estudiantes provenientes de las universidades buscan la manera de vincularse con el sector a través de algún proyecto de diseño o de consultorías universitarias financiadas por fondos de organismos gubernamentales, como Fondo Jalisco (Fojal); otras veces los estudiantes son obligados a realizar su servicio social o prácticas profesionales, aunque para esto último se firma un convenio marco que establece puntos principales para ambos.

Sobre la transferencia de conocimiento CTI hace falta mucho trabajo por hacer. Según los resultados, una buena parte del conocimiento se queda en las universidades y no se transfiere, por lo que se requiere impulsar este tipo de actividades para promover la industria mueblera jalisciense en mercados competitivos, con nuevos diseños, nuevas tecnologías, nueva infraestructura tecnológica de comercialización y nuevos perfiles de capital humano.

Los nuevos retos y desafíos de investigación son el involucramiento de organismos gubernamentales y organismos empresariales, así como las universidades para fortalecer la triple, cuádruple y quíntuple hélice con el sector industrial mueblero y transferir conocimiento científico, tecnológico y de innovación.

La siguiente línea de investigación es la transferencia de conocimiento CTI tomando en cuenta los indicadores internacionales de vinculación e innovación entre la academia y el sector mueblero sobre 
la realidad aumentada, nuevos recubrimientos, estructura multifuncionales y la ergonomía que se compite cada vez en países desarrollados.

\section{REFERENCIAS}

Ahonen, L. y T. Hämäläinen (2012), "CLIQ: A Practical Approach to the Quadruple Helix", en S. MacGregor \& T. Carleton, Sustaining Innovation; Collaboration Models for a Complex World. p. 191. London, Springer. $<$ doi https://doi.org/10.1007/978-1-4614-2077-4_2>.

Anderson, T.R., T.U. Daim \& F.F. Lavoie (2007). "Measuring the efficiency of university technology transfer", Science Direct: Technovation 27, pp. 306318. < doi https://doi.org/10.1016/j.technovation.2006.10.003>.

Arechavala, V.R. (2010), "Innovación Educativa, ¿en las universidades?", Concyteg, 20. <http://www.concyteg.gob.mx>.

Bautista, E.G. (2014), "La importancia de la vinculación universidad-empresagobierno en México", Revista Iberoamericana para la Investigación y el Desarrollo Educativo 7467, pp. 1-21. <https://www.ride.org.mx/index. $\mathrm{php} / \mathrm{RIDE} /$ issue/view/1>.

Bautista, E.G. (2015), "La vinculación entre agentes heterogéneos para la producción de conocimiento e innovación", Centro de Estudios e Investigación para el Desarrollo Docente AC, 24, 7467. <https://www.ride.org.mx/ index.php/RIDE/issue/view/1>.

Creswell, J.W. (2014), Research design; Qualitative, Quantitative, and Mixed Methods Approaches. EE.UU., SAGE.

D’Este, P. y S. Iammarino (2009), "The spatial profile of university-business research partnerships", Intangible Assets and Regional Economic Growth, pp. 1-30. <doi https://doi.org/10.1111/j.1435-5957.2010.00292.x>.

D’Este, P. y P. Patel (2005), "University - Industry linkages in the UK: what are the factors determining the variety of university researchers' interactions with industry?", The DRUID Tenth Anniversary Summer Conference, pp. 1-29. < doi https://doi.org/10.1016/j.respol.2007.05.002>.

D’Este, P., M.E. Castro y G.J. Molas (2014), Manual de Indicadores de Vinculación de la universidad con el entorno socioeconómico. Valencia, Ingenio. $<$ http://www.ricyt.org/2017/06/manual-de-indicadores-de-vinculacionde-la-universidad-con-el-entorno-socioeconomico-manual-de-valencia/>.

Debackere, K. y R. Veugelers (2005), "The role of academic technology transfer organizations in improving industry science links", Research Policy, pp. 321-342. < doi https://doi.org/10.1016/j.respol.2004.12.003>.

Drucker, E.P. (1986), Innovation and Entre-prenurship; practice and principles. EE.UU., Harper. <https://books.google.com.mx/books?id=M1NsLls R3hgC\&hl=es\&source=gbs_book_other_versions $>$.

Etzkowitz, H. y L. Leydesdorff (1995), "The Triple Helix. University-Indus- 
try-Government Relations: A Laboratory for Knowledge-Based Economic Development", EASST Review, 14, pp. 14-19. <https://papers.ssrn.com/ sol3/papers.cfm?abstract_id $=2480085>$.

García, L. L. (2004), "Estrategias de gestión para la capitalización del conocimiento en el contexto de la relación universidad. Sector productivo", Educere: Revista Venezolana de Educación, (27), pp. 507-516. <https:// dialnet.unirioja.es/servlet/articulo?codigo $=1148907>$

Geels, F.W. (2006), "Co-evolutionary and multi-level dynamics in transitions: The transformation of aviation systems and the shift from propeller to turbojet (1930-1970)", Technovation, 29(9) pp. 999-1016. <https://doi. org/10.1016/j.2005.08.010>.

Guerrero, M. y C. Santamaría (2020), "Ecosistema y actividad emprendedora en México: un análisis exploratorio", Perfiles Latinoamericanos, pp. $227-$ 252. <doi 10.18504/PL2855-009-2020>.

Hernández Sampieri, R., C. Fernández Collado y L. Baptista (2010), Metodología de la Investigación. México DF: McGraw Hill

Lai, W.H. (2011), "Willingness-to-engage in technology transfer in industryuniversity collaborations", Journal of Business Research in Elsevier, pp. 1218-1223. <doi https://doi.org/10.1016/j.jbusres.2011.06.026>.

Londoño, G.J., R.S. Velásquez, R.M. Villa, C.F. Franco y R.N. Viana (2018), "Identificación de tipos, modelos y mecanismos de transferencia tecnológica que apalancan la innovación", Revista CINTEX, 23 (2), pp. 13-23. <doi https://doi.org/10.33131/24222208.314>.

Lundvall, B.A. (1992), National Systems of Innovation: Towards a Theory of innovation and interactive learning. London, Pinter. < doi https://doi. org/10.1080/08109029308629360>.

Montiel, L.Y., B.J. Escobar, É.L. Castillo y C.A. González (2020), "El papel de la transferencia internacional de tecnología en los sistemas nacionales de innovación de los países en desarrollo", Revista científica ECOCIEN$C I A$, pp. 1-32. $<$ http://revistas.ecotec.edu.ec/index.php/ecociencia/article/ view/296>.

Nelson, R.R. (1993), National Innovation Systems:A comparative Study. Oxford, Oxford University Press. <https://papers.ssrn.com/sol3/papers. cfm?abstract id=1496195>.

Penprase, B.E. (2018), "The Fourth Industrial Revolution and Higher Education", en N.W. Gleason, Higher Education in the Era of the Fourth Industrial Revolution (1,) 238. Singapore, Yale-NUS College. $<$ https://link. springer.com/content/pdf/10.1007\%2F978-981-13-0194-0.pdf $>$.

Rickne, A., S. Laestadius y H. Etzkowitz (2013), "Innovation Governance in an Open Economy, Routledge: EE.UU.", Routledge. <doi https://doi. org/10.4324/9780203121306>.

Siegel, D., D. Waldman y A. Link (2003), "Assessing the impact of organizational practices on the relative productivity of university technology 
transfer offices: an exploratory study", Research Policy, pp. 27-48. <doi 10.1016/S0048-7333(01)00196-2>.

Touriñán, L.J. (2019), "La transferencia de conocimiento como proceso: de la universidad al sector educativa. una mirada desde la pedagogía", Resvista boletín REDIPE, pp. 20-65. <https://revista.redipe.org/index.php/1/ article/view/695>.

Viale, R. y H. Etzkowitz (2010), The Capitalization of Knowledge; A Triple Helix of University-Industry-Goverment. Cheltenham, UK / Northampton, MA, USA, Edward Elgar Publishing Limited. <https://www.worldcat. org/title/capitalization-of-knowledge-a-triple-helix-of-university-industry-government/oclc /1028008879? referer=di\&ht=edition>.

Wright, M., B. Clarysse, A. Lockett y M. Knockaert (2008), "Mid-range universities' linkages with industry: Knowledge types and the role of intermediaries", Research Policy 37, pp. 1205-1223. <doi https://doi.org/10.1016/j. respol.2008.04.021>. 